\title{
Contralateral Optic Neuritis Following Silicone Oil Endotamponade for Retinal Detachment
}

\author{
Patricio S Espinosa ${ }^{1,2,3}$, Patricio H Espinosa ${ }^{2,3}$, Charles D Smith ${ }^{4}$ and Joseph R Berger ${ }^{4}$ \\ ${ }^{1}$ Universidad San Francisco de Quito USFQ, Ecuador \\ ${ }^{2}$ Universidad Central del Ecuador, Ecuador \\ ${ }^{3}$ Centro Internacional en Neurociencias Quito, Ecuador \\ ${ }^{4}$ Department of Neurology of the University of Kentucky, Lexington, $K Y$
}

\begin{abstract}
Silicone oils are used for ocular endotamponade to treat complicated retinal detachments. Neurological complications of silicone oil endotamponade are uncommon. Case reports have documented optic nerve atrophy and migration of the silicone oil into the subarachnoid space and ventricular system. However, optic neuritis contralateral to the treated eye has not been previously described. We report a 60 year-old-woman who underwent repair of retinal detachment with silicone oil in the left eye and later developed optic neuritis in the right eye.

Immediately after the introduction of the silicone oil, the patient complained of severe pain in the left orbit with radiation into the left temporal and occipital areas. Subsequently, she developed vision loss in the previously unaffected right eye. She received a high dose intravenous steroids for three days with subsequent full recovery of vision in the right eye. Two weeks later, she had a second episode of decreased vision in the right eye, however this time, the vision in her right eye did not improve. MR imaging shows inflammation on the optic nerves and silicone oil in the ventricular system.
\end{abstract}

Conclusions: Silicone oil can migrate into the nervous system and may provoke optic neuritis in the contralateral eye.

\section{Introduction}

Silicone oils (polymethylsiloxanes) are used for ocular endotamponade to treat complicated retinal detachments [1]. Neurological complications of silicone oil endotamponade are uncommon. Case reports have documented optic nerve atrophy and migration of the silicone oil into the subarachnoid space and ventricular system [2-7]. However, optic neuritis contralateral to the treated eye has not been previously described. We report a patient that underwent placement of silicone oil in the left eye for retinal detachment who subsequently developed vision loss in the contralateral eye, There was accompanying radiographic evidence of silicone oil migration into the central nervous system.

\section{Case Report}

A 60 year-old-woman with a past medical history of hypothyroidism presented to the ophthalmologist with a left retinal detachment. Repair of the retinal detachment was attempted by placing silicone oil in this eye. Immediately after the introduction of the silicone oil, the patient complained of severe pain in the left orbit with radiation into the left temporal and occipital areas. This pain increased with eye movement and was refractory to analgesics. Fifteen days postoperatively, she developed vision loss in the previously unaffected right eye and ptosis on the left. She was admitted to hospital and treated with intravenous steroids for three days with subsequent oral prednisone taper. Four days after the hospitalization, she had full recovery of vision in the right eye and resolution of the left ptosis. The discharge diagnosis was optic neuritis. Two weeks later, she had a second episode of decreased vision in the right eye. Oral prednisone was prescribed as an outpatient. The vision in her right eye did not improve and she was referred for a second opinion at our clinic three months after the initial surgery.

On neurological examination, the right pupil measured $4 \mathrm{~mm}$ and was reactive to both light and accommodation. The left pupil was $4 \mathrm{~mm}$ and nonreactive. Visual acuity was $20 / 800$ in the right eye with normal visual fields and hand motion only in the left. Funduscopy of the right eye was normal. The silicone foreign body rendered funduscopy of the left eye impossible. There was no ptosis and ocular motility of both eyes was normal. The remainder of the neurological examination was normal.

Laboratory analysis including $\mathrm{CBC}$ with differential, routine chemistries, thyroid function tests and rheumatological screen (ANA, ANCA, Anti DNA) were normal or negative. CSF analysis showed 20 white blood cells $/ \mathrm{mm}^{3}$ (83\% monocytes and $17 \%$ polymorphonuclear cells), $1 \mathrm{red}$ blood cell $/ \mathrm{mm}^{3}$, glucose $75 \mathrm{mg} / \mathrm{dl}$, and protein $26 \mathrm{mg} /$ dl. CSF cytology was negative for malignancy. CSF Gram stain and and cultures were negative. Pattern reversal visual evoked potentials showed no evoked responses in the left eye and a markedly delayed $\mathrm{P} 100$ in the right eye $(221 \mathrm{~ms})$.

MRI imaging performed $\sim 40$ days after the initial endotamponade showed inflammation around the orbit, menigeal ehacement adyacent to the orbit in the left temporal region, and hypointense signal in the optic nerves more noticible in the left. (Figure 1a and Figure 1b) FLAIR axial images showed a round hyperintense signal in the left lateral ventricle with the same intensity as the contents of the left eye consistent with silicone oil (Figure 1c).

*Corresponding author: Dr. Patricio S. Espinosa, Centro Internacional en Neurociencias, 5 Finch Ln, Mandeville, LA 70471, E-mail: ps.espinosa@cienecuador.org

Received February 03, 2011; Accepted April 08, 2011; Published April 10, 2011

Citation: Espinosa PS, Espinosa PH, Smith CD, Berger JR (2011) Contralateral Optic Neuritis Following Silicone Oil Endotamponade for Retinal Detachment. J Neurol Neurophysiol 2:112. doi:10.4172/2155-9562.1000112

Copyright: (c) 2011 Espinosa PS, et al. This is an open-access article distributed under the terms of the Creative Commons Attribution License, which permits unrestricted use, distribution, and reproduction in any medium, provided the original author and source are credited. 
Citation: Espinosa PS, Espinosa PH, Smith CD, Berger JR (2011) Contralateral Optic Neuritis Following Silicone Oil Endotamponade for Retinal Detachment. J Neurol Neurophysiol 2:112. doi:10.4172/2155-9562.1000112

\section{Discussion}

Silicone oil migration into the nervous system is an uncommon complication but has been reported in few instances (Table 1). Migration of silicone oil from the vitreous cavity into the ipsilateral optic nerve has been well documented [8]. However, optic neuritis of the contralateral eye has not been reported. We hypothesize that reason for the vision loss in the contralateral eye of the silicone oil endotamponate was do to an inflammatory reaction in the optic nerve. The clinical presentation of rapid visual, retro-orbital and periorbital pain evoked by eye movement, response to steroids, and evoked potential strongly suggest a retro-orbital optic neuritis. The hypointense signal abnormalities on MRI seen in both optic nerves, more on the left, suggest the presence of silicone. There are two pathogenetic possibilities: A) the silicone oil migrated trough the optic nerve to the chiasm to the contralateral optic nerve and caused demyelination/inflammation that caused blindness and/or B) the silicone oil that migrated to the ipsilateral optic nerve made the optic nerves immunologically attractive (antigen presentation) to the immune system causing demyelination of the optic nerves.

It is uncertain how silicone oil accesses the nervous system. The silicone could migrate from the retina to the optic nerve and later into the subarachnoid space that surrounds the optic nerve eventually entering traveling into the intracranial subarchnoid space and thence trough the foramens of Lushka into the ventricular system (Figure 1b). An alternative hypothesis is that silicone oil was absorbed trough the surrounding orbital venous system in the eye, then into the blood stream and ultrafiltered with the CSF into the lateral ventricles and later into the subarchnoid space. However, with respect to the latter hypothesis, neither we nor others have found evidence of silicone oil seeding other organs.

The immune response to the silicone oil is mediated by monocytes [9]. Silicone vacuoles have been found on microscopic examination of retinal tissue and optic nerve $[9,10]$. We do not have a tissue sample in our patient, however the CSF analysis showed pleocytosis with the majority of cells being monocytes (87\%). This finding may argues in favor that an immune response was triggered in the CNS by the silicone oil.

Ptosis has been reported as consequence of silicone oil intrusion in the upper eyelid in patients with retinal detachment due to a lipogranulomatous inflammation $[11,12]$. Our patient shows increased fat signal around the left which can correlate with inflammation of this region due to the presence of silicone which was lessen by the intravenous and oral corticosteroids. Another possibility is that the oculomotor nerve was injured or compressed as a result of the surgery and surrounding inflammation; however, the rapid resolution of the ptosis after intravenous corticosteroids makes this less likely.

Neuromyelitis optica (NMO) antibody was not tested due to

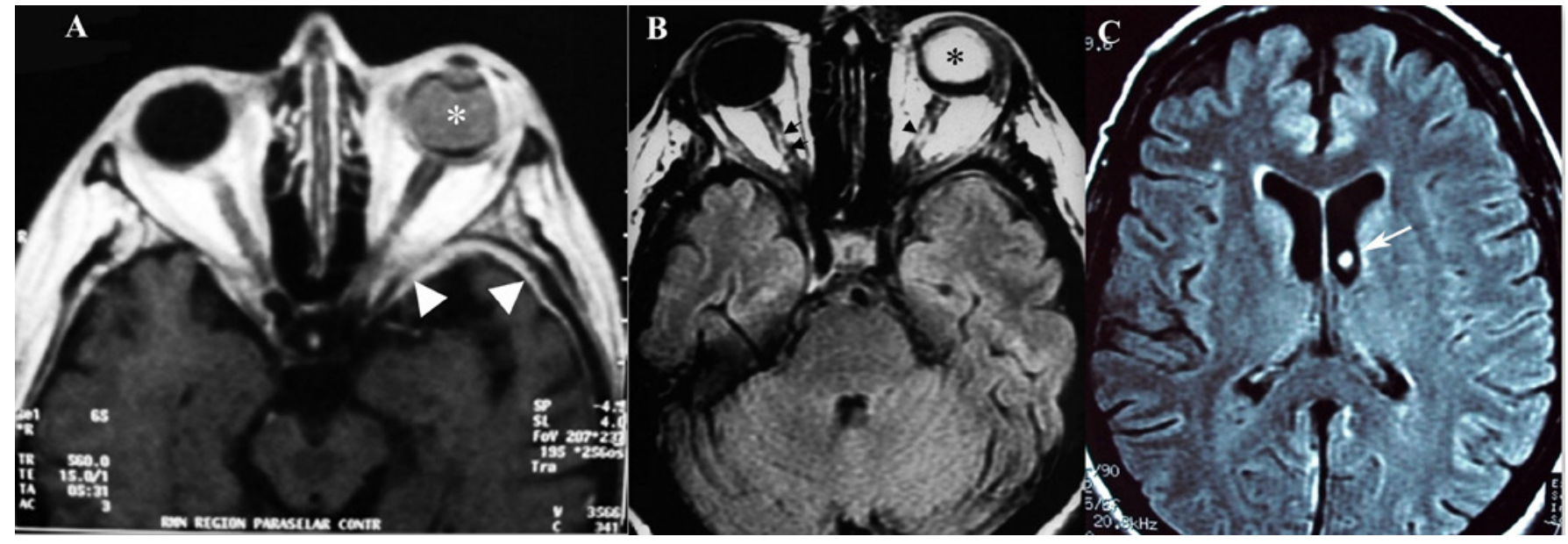

Figure 1: (A) T1 weighted axial image with contrast shows: (a) hyperintense signal in the left eye consistent with the silicone oil placed for the retinal detachment (white asterisk), b) meningeal enhancement in the left temporal pole region (white arrowheads). (B) MRI FLAIR image shows: (a) hyperintense signal within vitreal cavity representing silicone oil in the left eye (black asterisk), and (b) hyperintense signal in the optic nerves on the right (double black arrows) and left (single black arrowhead), indicating inflammation and/or edema. (C) Round hyperintense signal in the left lateral ventricle with the same intensity as the contents of the left eye consistent with silicone oil (white arrow)

\begin{tabular}{|c|c|c|c|}
\hline Reference & Age & Gender & Neurological Complications \\
\hline Espinosa et al. 2009 Current case & 64 & Woman & $\begin{array}{l}\text { Vision loss in the contra lateral eye ( suspected optic neuritis), ipsilateral ptosis, chemical local } \\
\text { meningitis, migration of silicone oil into sella turcica subarchnoid space and ventricular system. }\end{array}$ \\
\hline Fangtian et al. 2005 & 62 & Woman & $\begin{array}{l}\text { Episode of loss of consiuness for five minutes ( possible seizure), Migration of silicone into the } \\
\text { sella turcica and to the ventricular system }\end{array}$ \\
\hline Yu et al. 2005 & 47 & Man & Change in mental status and silicone oil in the subarchnoid space and ventricular system. \\
\hline Eller et al. 2000 & 42 & Woman & Migration of silicone oil into the subarchnoid space and ventricular system. Patient HIV (+) \\
\hline Kuhn et al. 2000 & 15 & Woman & $\begin{array}{l}\text { Severe headaches, worsening vision in the ipsilateral eye to the silicone oil placement and } \\
\text { migration of silicone oil into the subarchnoid space and ventricular system }\end{array}$ \\
\hline Williams et al. 1999 & 42 & Man & Migration of silicone oil into the subarchnoid space and ventricular system. Patient HIV (+) \\
\hline
\end{tabular}

Table 1: Reported Neurological complications of silicone oil endotamponade. 
Citation: Espinosa PS, Espinosa PH, Smith CD, Berger JR (2011) Contralateral Optic Neuritis Following Silicone Oil Endotamponade for Retinal Detachment. J Neurol Neurophysiol 2:112. doi:10.4172/2155-9562.1000112

Page 3 of 3

logistic and economic difficulties, although it is unlikely that this was illness was the result of NMO. She was previously healthy patient and did not show any signs of myelitis.

\section{Conclusions}

Silicone oil can migrate into the nervous system and may provoke optic neuritis. Clinicians should be familiar with the neurological complications of silicone oil endotamponate. Signs such as refractory ocular pain, headache, ptosis, change in mental status, worsening vision loss (ipsilateral and/or contralateral) should raise the possibility of migration of silicone oil into the central nervous system.

\section{References}

1. Heimann H, Stappler T, Wong D (2008) Heavy tamponade 1: a review of indications, use, and complications. Eye 22: 1342-1359.

2. Fangtian D, Rongping D, Lin Z, Weihong $Y$ (2005) Migration of intraocular silicone into the cerebral ventricles. Am J Ophthalmol 140: 156-158.

3. Eller AW, Friberg TR, Mah F (2000) Migration of silicone oil into the brain: a complication of intraocular silicone oil for retinal tamponade. Am J Ophthalmol 129: 685-688.

4. Kuhn F, Kover F, Szabo I, Mester V (2006) Intracranial migration of silicone oil from an eye with optic pit. Graefes Arch Clin Exp Ophthalmol 244: 1360-1362.

5. Papp A, Toth J, Kerenyi T, Jackel M, Suveges I (2004) Silicone oil in the subarachnoidal space--a possible route to the brain? Pathol Res Pract 200: 247-252.

6. Yu JT, Apte RS (2005) A case of intravitreal silicone oil migration to the central nervous system. Retina 25: 791-793.

7. Williams RL, Beatty RL, Kanal E, Weissman JL (1999) MR imaging of intraventricular silicone: case report. Radiology 212: 151-154.

8. Taherian K, Bishop F, Woon WH, Nelson M (2004) Silicone oil endotamponade is it safe? Eye 18: 649-650.

9. Wickham L, Asaria RH, Alexander R, Luthert P, Charteris DG (2007) Immunopathology of intraocular silicone oil: enucleated eyes. $\mathrm{Br} \mathrm{J}$ Ophthalmol 91: 253-257.

10. Biswas J, Verma A, Davda MD, Ahuja S, Pushparaj V. (2008) Intraocular tissue migration of silicone oil after silicone oil tamponade: a histopathological study of enucleated silicone oil-filled eyes. Indian J Ophthalmol 56: 425-428.

11. Donker DL, Paridaens D, Mooy CM, van den Bosch WA (2005) Blepharoptosis and upper eyelid swelling due to lipogranulomatous inflammation caused by silicone oil. Am J Ophthalmol 140: 934-936.

12. Quintyn JC, Genevois O, Ranty ML, Retout A (2003) Silicone oil migration in the eyelid after vitrectomy for retinal detachment. Am J Ophthalmol 136: 540-542. 